\title{
New coherent detector for terahertz radiation based on excitonic electroabsorption
}

\author{
W. Sha and T. B. Norris \\ Center for Ultrafast Optical Science, University of Michigan, 2200 Bonisteel, Ann Arbor, \\ Michigan 48109-2099
}

J. W. Burm, D. Woodard, and W. J. Schaff

School of Electrical Engineering, Cornell University, Ithaca, New York, 14850

(Received 11 March 1992; accepted for publication 31 July 1992)

\begin{abstract}
We demonstrate a new technique for the coherent measurement of free-space THz electrical transients, based on the parallel-field excitonic electroabsorption effect in GaAs quantum wells. A THz transient generated from a photoconductive dipole antenna is measured with a rise time of $290 \mathrm{fs}$ and a full width at half maximum of $360 \mathrm{fs}$. The initial rise of the THz wave form is abrupt, and does not display the exponential leading edge apparent in waveforms measured with photoconductive techniques. The detector sensitivity is sub- $100 \mathrm{mV} / \mathrm{cm}$.
\end{abstract}

Coherent detection of free-space $\mathrm{THz}$ electrical transients has been based on two techniques: photoconductive gating of a dipole antenna ${ }^{1,2}$ or interferometric autocorrelation. ${ }^{3}$ Interferometric autocorrelation characterization is, in principle, limited only by the width of the THz. pulse itself (and thus, ultimately by the optical pulse width), and has been demonstrated with a time resolution of $140 \mathrm{fs}^{3}$ However, deconvolution of the autocorrelation requires some kind of assumption about the waveform of the transient, so the $\mathrm{THz}$ wave form is not resolved directly.

In photoconductive sampling, materials with a short photoconductivity lifetime, such as ion-implanted siliconon-sapphire or GaAs grown by molecular beam epitaxy at low temperatures (LT GaAs), are used as a gate to sample $\mathrm{THz}$ electrical transients. Due to its superior sensitivity, photoconductive sampling has been the main technique for measuring $\mathrm{THz}$ radiation pulses generated by femtosecond laser pulses. However, since this technique measures the detector photocurrent, which is the convolution of the $\mathrm{THz}$ electrical transient with the material photoconductivity response, its resolution is limited by the finite carrier lifetime (typically $>0.5 \mathrm{ps)}$ ) and by the complex transient conductivity response of carriers photogenerated by femtosecond pulses. ${ }^{46}$ In fact, although fast temporal features (as short as $190 \mathrm{fs}$ ) on the THz pulse can be resolved due to the fast rise of the detector photoconductivity (a point noted in Ref. 7), the exponential decay of the detector photoconductivity is manifested as a much slower component on the leading edge of the observed $\mathrm{THz}$ wave form. Clearly the asymmetrical gating function of the photoconductive detector results in a distortion of the $\mathrm{THz}$ transient.

For some applications, such as investigations of $\mathrm{THz}$ pulse generation mechanisms by both optical rectification $^{8,9}$ and carrier acceleration in strong fields, ${ }^{6,10,11}$ it is desired to measure the shape of the $\mathrm{THz}$ waveform as accurately as possible, with as high a time resolution as possible. In this letter, we describe a detector which utilizes the excitonic electroabsorption effect in a GaAs multiplequantum well (MQW) structure to detect the timedependent electric field of a free-space $\mathrm{THz}$ pulse. With this detector, we have been able to observe a $\mathrm{THz}$ radiation transient with a rise time of 290 fs (10\%-90\%) and a full width at half-maximum (FWHM) of $360 \mathrm{fs}$, and for the first time have observed the abrupt turn on of the $\mathrm{THz}$ pulse.

When an electric field is applied in the plane of a quantum well, the exciton transitions are broadened due to field ionization of the excitons. ${ }^{12}$ This change in the absorption, called the excitonic electroabsorption effect (EEE), may be probed with a short optical pulse. Thus, a time-dependent electric field in the quantum well plane may be measured with a time resolution that is limited, in principle, only by the exciton linewidth. ${ }^{13}$ Knox and coworkers have used the EEE to measure transient electric fields on various transmission line struclures, and have demonstrated rise times as short as $180 \mathrm{fs} .{ }^{14.15}$ Our work represents the extension of this technique to the measurement of electrical transients propagating in free space ( $\mathrm{THz}$ radiation). Of course, the free-space propagating field strengths are typically quite small $(\leqslant 10 \mathrm{~V} / \mathrm{cm})$, and the EEE response is vanishingly small for such fields. ${ }^{12}$ However, if the MQW structure is biased with a de field of about $10 \mathrm{kV} / \mathrm{cm}$, a THz field of 10 $\mathrm{V} / \mathrm{cm}$ across the gap will produce a modulation of the probe beam of up to $10^{-4}$, which is detectable using lock-in techniques.

The detector design is shown in Fig. 1. All the layers were grown on a semi-insulating $\mathrm{GaAs}$ substrate by molecular-beam epitaxy. First, an $\mathrm{Al}_{0.9} \mathrm{Ga}_{0.1} \mathrm{As} /$ $\mathrm{Al}_{0.35} \mathrm{Ga}_{0.65}$ As multilayer dielectric high-reflector stack was grown, followed by the MQW structure for EEE, which consists of 40 periods of nominally $51 \AA / 150 \AA$ $\mathrm{GaAs} / \mathrm{Al}_{0.3} \mathrm{Ga}_{0.7} \mathrm{As}$. The detector works as a reflectionmode absorption modulator, ${ }^{16}$ as illustrated in Figs. 1 and 2. A pair of electrodes is fabricated on the top of the sample to form a horizontal $p-i-n$ structure with a gap of 50 $\mu \mathrm{m}$. The dc electric field required to maximize the detector sensitivity is provided by a $40 \mathrm{~V}(8 \mathrm{kV} / \mathrm{cm})$ reverse bias to the $p-i-n$ structure, and the detector response is linear to small changes of the electric field.

The pump and probe pulses are generated by a 76$\mathrm{MHz}$ homemade self-mode-locked Ti:sapphire laser which produces $75-\mathrm{fs}$ pulses at $808 \mathrm{~nm}$ (which is at the peak of 


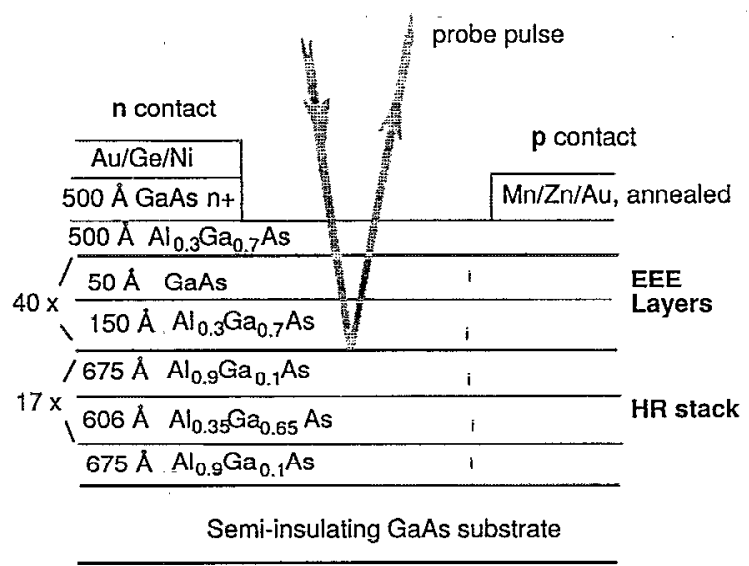

FIG. 1. Detector layer structure showing the high-reflector dielectric stack, the GaAs quantum wells used for excitonic electroabsorption, and the horizontal $p-i-n$ structure.

the detector heavy-hole exciton absorption), with a bandwidth of $14 \mathrm{~nm}$. An illustration of the $\mathrm{THz}$ generation and detection scheme is given in Fig. 2. The $\mathrm{THz}$ radiation is generaled by the pump beam incident on a biased LTGaAs photoconductive dipole antenna. The pump is chopped at $810 \mathrm{kHz}$ by an acoustic-optic modulator. (Broadening of the pulse due to group velocity dispersion in the modulator is compensated using a prism pair.) A pair of high resistivity silicon lenses is used for collimating the $\mathrm{THz}$ radiation from the transmitter and refocusing it to the detector. The variable aperture serves as a frequency filter, as will be discussed below. The $\mathrm{THz}$ transient field at the detector is sampled by an optical probe (gale) pulse with variable delay with respect to the pump pulse. The weak probe beam ( $1 \mathrm{~mW}$ ) is focused to a $30 \mu \mathrm{m}$ spot in the detector gap. The bandwidth of the probe is restricted to the exciton linewidth (at the dc bias field of $8 \mathrm{kV} / \mathrm{cm}$ ) using a 10-nm interference filter. An unmodulated reference beam and the reflected probe beam are detected by a pair of photodiodes (EG\&G FND-100), and the diode outputs are fed into a differential preamplifier (EG\&G 113). The amplified signal at $810 \mathrm{kIIz}$ is mixed with a synchronized local oscillator at $800 \mathrm{kHz}$ (from a two-

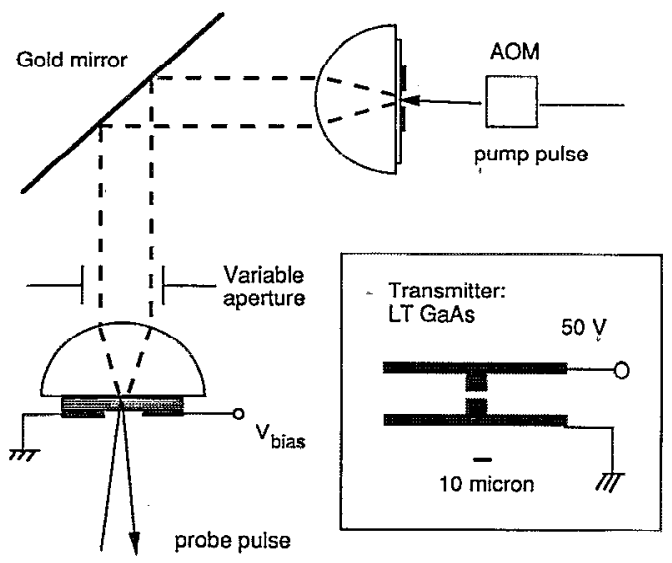

FIG. 2. Schematic of the THzpulse generation and detection.

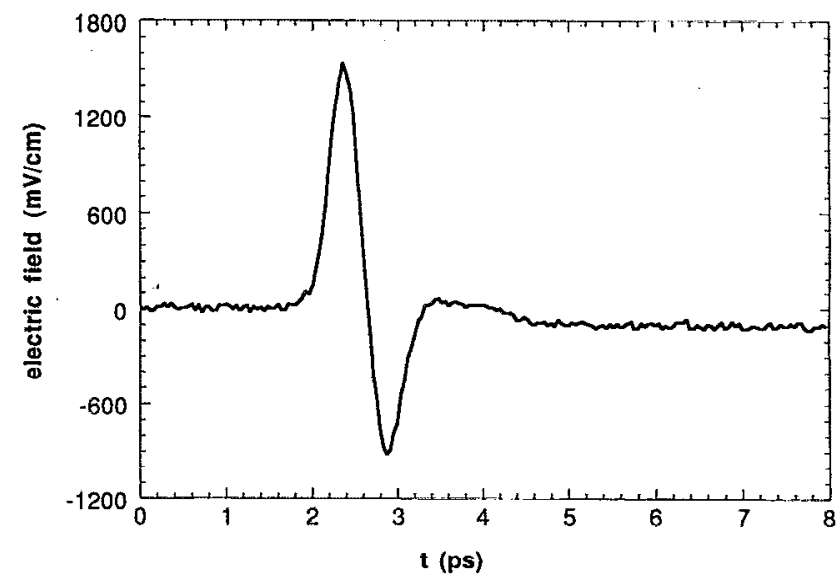

FIG. 3. TIIz waveform measured by the electroabsorption delector.

channel HP3326-A synthesizer), and the down-converted signal at the difference frequency (IF) $10 \mathrm{kHz}$ is measured by a $Q=100$ audiofrequency lock-in amplifier (EG\&G 124A). By modulating the pump at a high frequency where the laser noise is minimized, ${ }^{17}$ and mixing the signal down to the lock-in amplifier, a detection sensitivity of better than $10^{-6}$ can be attained. ${ }^{18}$

Shown in Fig. 3 is a $\mathrm{THz}$ pulse waveform sampled by the electroabsorption detector. The aperture is set at a diameter of $5 \mathrm{~mm}$ to filter out trailing low frequency components. The trace is acquired using a 100-ms integration time on the lock-in and 50 scans of averaging. It shows a $10 \%-90 \%$ rise time of 290 fs and a FWHM of 360 fs. More importantly, the $\mathrm{THz}$ transient has an abrupt turn on, and does not show the exponential tail on the leading edge of the waveform that is generally observed with photoconductive detection. The electric field can be easily and accurately calibrated by the detector response to a small ac voltage applied to the gap; we find the electric field at the peak of the waveform is $1.5 \mathrm{~V} / \mathrm{cm}$ and the noise floor is below $100 \mathrm{mV} / \mathrm{cm}$. We believe that the detection sensitivity could be further improved with an antireflection coating on the detector (to eliminate Fabry-Perot effects) and optimization of the electronics.

A comparison of the electroabsorption detector with conventional photoconductive detection (using a LTGaAs dipole antenna) is given in Fig. 4. The same transmitter is used, and the results for a series of aperture openings are shown. Each detector is positioned to maximize the peak amplitude of the $\mathrm{THz}$ pulse. Because we find that the measured waveform is very sensitive to detector alignment, one cannot compare the waveforms in too great detail. Nevertheless, certain qualitative conclusions can be readily drawn. With the electroabsorption detector, the onset of the $\mathrm{THz}$ radiation is clearly seen as a fast rising edge which basically remains the same for different aperture openings, and only the following low frequency components are altered by the aperture. With the photoconductive detector, the exponential leading edges are associated with the material lifetime. Due to the convolution of the $\mathrm{THz}$ pulse with the asymmetrical gate given by the transient photoconductivity of the detector antenna, the wave- 

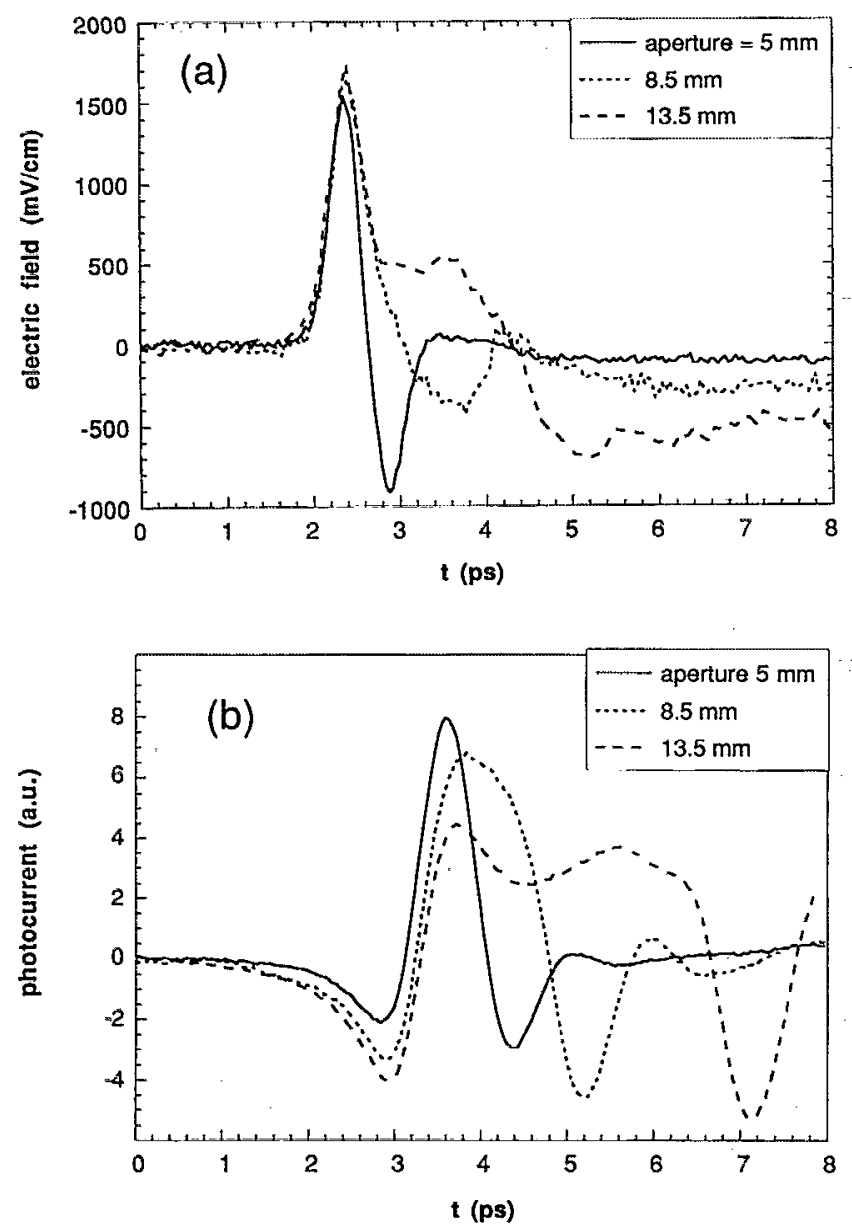

FIG. 4. A comparison of $\mathrm{I} \mathrm{Hz}$ waveforms measured by both (a) electroabsorption, and (b) photoconductor detection, for various aperture sizes.

form changes dramatically with different aperture openings and the abrupt onset of the $\mathrm{THz}$ radiation is not seen.

It should be noted that the resolved waveform with 290 fs rise time may be the limit of the transmitter instead of the limit of the time resolution of the electroabsorption detector. In fact, for a bias field of $8 \mathrm{kV} / \mathrm{cm}$ on the detector, a time resolution of closer to $100 \mathrm{fs}$ is expected. ${ }^{13} \mathrm{An}$ ultimate test of the speed of the detector could be the use of an optical rectification process, such as the inverse franzKeldysh effect ${ }^{8,9}$ in which the $\mathrm{THz}$ electrical transient is as short as the optical pulse.
In conclusion, we demonstrate a new coherent detector for $\mathrm{THz}$ radiation measurement using the excitonic electroabsorption effect in quantum wells. A $\mathrm{THz}$ pulse with $290 \mathrm{fs}$ rise time and $360 \mathrm{fs}$ FWHM is resolved which is substantially shorter than that probed by photoconductive sampling with the same transmitter. The ultimate time resolution of this detector is expected to be shorter than 100 fs.

The authors would like to acknowledge helpful discussions with G. Mourou and T. Motet. They are grateful for technical assistance from T. Motet and D. Craig. This work was supported by the AFOSR under Grant No. URI-090-0214, and by the NSF through the Center for Ultrafast Optical Science under STC PHY 8920108.

${ }^{1}$ P. R. Smith, D. H. Auston, and M. C. Nuss, IEEE J. Quant. Electronn. QE-24, 255 (1988), and references therein.

${ }^{2}$ M. V. Exter and D. R. Grischkowsky, IEEE Trans. Micowave Theory Tech. MTT-38, 1684 (1990).

${ }^{3}$ B. I. Greene, J. F. Federici, D. R. Dykaar, R. R. Jones, and P. H. Bucksbaum, Appl. Phys. Lett. 59, 893 (1991).

${ }^{4}$ M. C. Nuss, D. H. Auston, and F. Capasso, Phys. Rev. Lett. 58, 2355 (1987).

${ }^{5}$ A. S. Vengurlekar and S. S. Jha, Phys. Rev. B 38, 2044 (1988).

${ }^{6}$ W. Sha, J. Rhee, T. B. Norris, and W. J. Schaff, IEEE J. Quant. Electron., special issue on Ultrafast Optics and Optoelectronics, Oct. 92 ( to be published).

${ }^{7}$ N. Katzenellenbogen and D. Grischkowsky, Appl. Phys. Lett. 58, 222 (1991).

${ }^{8}$ B. B. Hu, X.-C. Zhang, and D. H. Auston, Phys. Rev. Lett. 67, 2709 (1991).

${ }^{9}$ Shun Lien Chuang, S. Schmitt-Rink, B. I. Greene, P. N. Saeta, and A. F. J. Levi, Phys. Rev. Lett. 68, 102 (1992).

${ }^{10}$ W. Sha, T. B. Norris, W. J. Schaff, and K. E. Meyer, Phys. Rev. Lett. 67, 2553 (1991).

${ }^{11}$ X.-C. Zhang, B. B. Hu, J. T. Darrow, and D. H. Auston, Appl. Phys. Lett. 56, 1011 (1990).

${ }^{12}$ D. A. B. Miller, D. S. Chemla, T. C. Damen, A. C. Gossard, W. Wiegmann, T. H. Wood, and C. A. Burrus, Phys. Rev. B 32, 1043 (1985).

${ }^{13}$ S. Schmitt-Rink, D. S. Chemla, W. H. Knox, and D. A. B. Miller, Opt. Lett. 15, 60 (1990).

${ }^{14}$ W. H. Knox, D. A. B. Miller, T. C. Damen, D. S. Chemla, C. V. Shank, and A. C. Gossard, Appl. Phys. Lett. 48, 864 (1986).

${ }^{15}$ W. H. Knox, J. E. Henry, K. W. Goossen, K. D. Li, B. Tell, D. A. B. Miller, D. S Chemla, A. C. Gossard, J. English, and S. Schmitt-Rink, IEEE J. Quantum. Electron. QE-25, 2586 (1989).

${ }^{16}$ G. D. Boyd, D. A. B. Miller, D. S. Chemla, S. L. McCall, A. C. Gossard, and J. H. English, Appl. Phys. Lett. 50, 1119 (1987).

${ }^{17}$ J. Son, J. V. Rudd, and J. F. Whitaker, Opt. Lett. 17, 733 (1992).

${ }^{18}$ J. M. Chwalek and D. R. Dykaar, Rev. Sci. Instrum. 61, 1273 (1990). 